\title{
ASSESSMENT OF FLEXIBLE FEATURES IN THE DESIGN OF EVENT CENTRES IN MINNA, NIGER STATE NIGERIA
}

\author{
P. Ayuba ${ }^{1, *}$ and F. A. Agah ${ }^{2}$

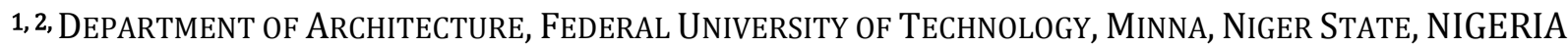 \\ E-mail addresses: ${ }^{1}$ arcayubaxx2@futminna.edu.ng, 2 agahfaith09@gmail.com
}

\begin{abstract}
Flexibility overtime has been known to enhance the peculiarity of a space, making spaces adaptable to multipurpose use, without altering the functional requirements. The design features and techniques that allow for changing situations in the use and operation of spaces are the focus of this paper. Components such as walls, floors and roofs are the design features that affect space in any building. Event centres being hubs for cultural and social activities, attract an unpredictable population, hence the need for flexible venues for event centres which are fast becoming stable and developing features of any city. The study assessed available event centres and the design features used to achieve flexibility, examining their adaptation to inadequacy of event spaces in selected event centres in Minna Niger state. Observation schedules was used as instrument to obtain data, the data collected was then analysed using Microsoft excel. It was observed from the study that all event centres had adaptability as their flexible design approach and this is as a result of the use of large open spaces, however $80 \%$ of event centres in Minna, Niger state failed to have flexible design features. The $20 \%$ that provided few of these features used curtain as flexible design elements. The paper recommended that design features for flexibility such as moveable walls, retractable roofs and sitting should be used in event centre designs to achieve an effective flexible design.
\end{abstract}

\section{Keywords: Adaptation, Design features, Event centre, Flexibility}

\section{INTRODUCTION}

Architecture is about concepts and design decisions made to enable effective satisfaction of a software system's quality requirements [1]. The degree to which a system supports possible or future changes to its requirements is what is known as flexibility [2]. Flexible spaces could be identified and restricted through design features such as floors, ceilings and walls and these should be designed in a way that changes flexibly. A space may be required to be shrunk or expanded and since these design features identify and restrict the space, they should be designed for dissociation or separation to achieve a flexible space. An event centre offers a multipurpose space where events with a huge amount of people take place like exhibition halls, conference centres, exposition centres and convention centres. Flexibility and adaptability are key factors required in these spaces in order to be subdivided into various sized spaces as the case may be. These sizes expand the design possibilities for demountable structures using modular construction. Each module can be attached, detached, modified, relocated and replaced easily for upgrading, repair, recycling and reuse. The importance of Minna has further been enhanced with the movement of the seat of Federal Government from Lagos to Abuja in 1992. Minna is about $120 \mathrm{~km}$ to the west of Abuja; it is the closest state capital to the Federal Capital Territory. Since then both the population of Minna and its economic land space have impact on spatial extent of the town.

\section{LITERATURE REVIEW}

\subsection{Design Features in a Building Structure}

Design features are the distinguishing characteristics or unique component parts that, together, form the houses, buildings and structures. A building includes the structure itself and the non-structural components attached to and supported by the structure. For a basic structure, the walls, floors and roofs make up these features and components [3]. 


\subsubsection{Walls}

Walls are vertical structural elements used to divide or enclose and in building construction, to form boundary of a room or a building. They are the physical barriers that provide privacy, security, and fire and sound separation. In traditional masonry construction, they support the weight of floors and roofs but modern steel and reinforced concrete frames, as well as heavy timber and other skeletal structures, require exterior walls only for shelter and sometimes distribute with them on the ground floor to permit easier access [4]. Positioning of walls depend on type of support given floor and roofs. These walls can be categorised according to their functions; load bearing walls and non-load bearing walls. Load bearing walls are part of the structure of a building, that bear a load resting upon it by conducting its weight to a foundation structure, and their maximum length establishes the distance between them [5]. Materials often used to construct load bearing walls in large buildings are concrete, blocks or bricks. Non-bearing walls are partition walls that are used to divide rooms, used where loads are carried by beams, or other members, and are sometimes referred to as curtain walls; they are attached to the frame members. Any durable, weather-resisting material such as glass, plastic, metal alloy, or wood may be used, since non-bearing walls are freed from the limitations of structural requirements [4].

\subsubsection{Floors}

Floor is regarded as the lower enclosing and supporting surface that extends horizontally throughout a structure. It forms the bottom of a room and divides space horizontally into stories. Floor systems form a horizontal partition at each level where they occur and transfer load to braced walls below that floor level or directly to the foundation when the lowest floor is supported on a foundation. The floor assembly must support its own dead load plus furnishings and the live load of occupants engaged in any variety of activities [3]. The horizontal supports beneath its top surface-and the vertical supports with which they intersect to form a frame must be sufficiently large and spaced closely to prevent sagging of the assembly. Materials used for floors range from compacted soil to reinforced concrete [5].

\subsubsection{Roof}

Roof is the covering of the top of a building, serving to protect against rain, snow, sunlight, wind and extremes of temperature. In many buildings, the roof is a major element that gives the building its characteristic profile [6]. Roofs have been constructed in a variety of forms as dictated by technical, economic or aesthetic considerations.

\subsection{Overview of Flexibility}

Humans are flexible beings and the survival of humans as a species was due to man's capacity of changing, to be adaptable and movable. Man's curiosity to explore and accomplish new goals showed that humans are dynamic and have dynamic behaviours [7]. A simple cave could be enlarged, changed in shape, have another chamber added to it behind, to one side, above or below, linked by ramp, stair or doorway, and then another chamber beyond that, perhaps, in a different direction, or branching off from one of the new chambers, thereby adapting to existing needs [8].

In 1914, Domino house was designed using the principle of "Plan Libre" as seen in Figure 1 which was one of the quintuple principles proposed by Le Corbusier modern architecture, in the early 20th century. The plan was an open design, having no internal walls except for space partitioning, column supports and stairs to connect levels together. Internal walls could be placed wherever required so that spaces can be shrunk or expanded, this idea offered freelance in designing the spaces and bringing flexibility for spaces.

Flexibility has inspired creativity worldwide due to its outstanding characteristics of adaptability, mobility, and transformability. It is an important concept that has entered contemporary architectural world and this is because it brings about the possibilities of portable, prefabricated, demountable, dynamic, adaptable, mobile structures and thereby rapidly becoming a trend in modern day architecture.

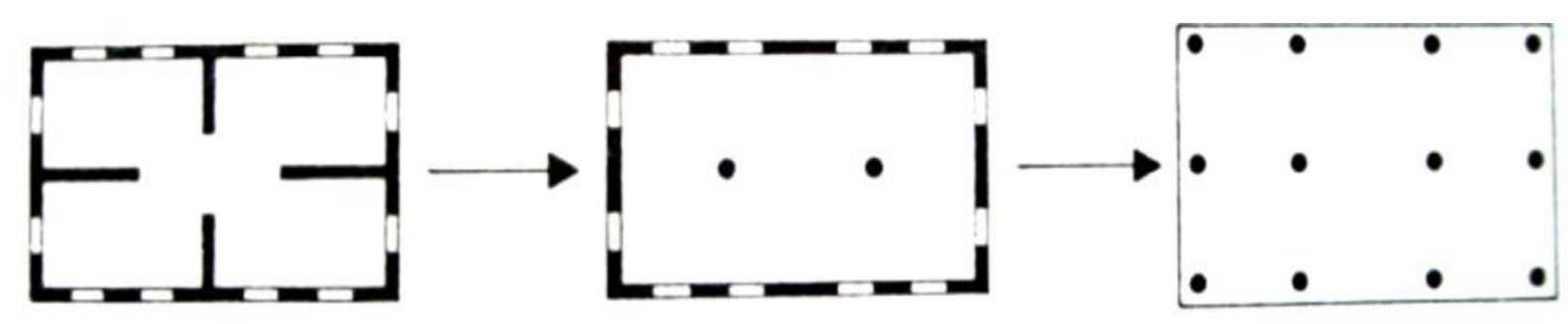

Figure 1: The idea of Plan Libre (Source [19]) 
Buildings no longer represent a static categorised order: instead, they have become flexible containers for use by a constantly changing society. Flexible buildings are intended to respond to changing situations in their use, operation and location [7]. They can evolve from a reality to another and accommodate new purposes and needs and the possibilities are vast and limitless. Flexibility means it is not static, rather, it is movable, adapts to change and transforms [9].

\subsection{Flexibility in the Design of Buildings}

Flexibility in the design of buildings is adapting a concept where buildings can adapt to current and future needs ranging from economic concerns, to sustainability and the possibility of change and also allows the division of the space according to the needs of the user. Flexibility in buildings is designing for changing requirements and arrangements [10]. In order for a building design to be flexible, the following must be considered: adaptability, mobility and transformability.

\subsubsection{Adaptability}

Every building should be designed to have the ability to alter to changes or be changed to fit current circumstances. In architecture, change is inevitable therefore having a building with elements that allow for change to happen is the major concern so that while a building has a distinct purpose, it can operate as multipurpose [11]. A space capable of meeting different requirements by simply changing of furniture, without any further modification can be seen as an adaptable space. The open building approach considered the most formalized approach for adaptable architecture. The flexibility of the building design allows for users and occupants to choose own designer, and freedom for the designer to create the desired space the client needs. An example of adaptable building is the Schlaulager (Art Store) in Basel as seen in Plate I(b) showing an open space which combines storage, exhibition spaces and art research institute. The storage concept ensures that works remain accessible unlike in a typical museum situation where art not on display is crated up and locked in a vault. Plate I(a) shows the exterior view of the Art Store.

\subsubsection{Mobility}

Mobility of buildings represent the physical movement of a building that changes places within a time range. Relocation according to specific needs is the basic idea behind mobile structures all over the world. This was practised by the nomadic communities who take their dwellings with them [11]. Mobile structures are usually demountable buildings that promote movement and flexibility of space, widely used in a number of fields such as in commerce, industry, military, education, health care, housing, where they fulfil their individual roles [12]. Flexibility in the design of buildings, using mobile design is related with the possibility of designing temporary accommodations in critical and emergency situations such as in war and conflict areas or in relation to natural disasters. Mobility of buildings gives rise to efficient use of materials and resources making it important for flexibility. Plate II is an example of a mobile structure; the Halley VI Antarctica Research Station that served as a home and work station for 50 scientists, designed by London-based Hugh Broughton Architects.

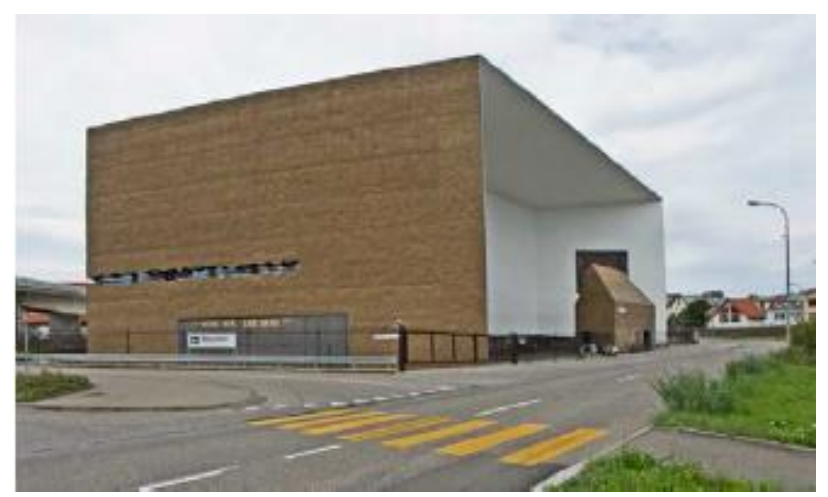

(a)

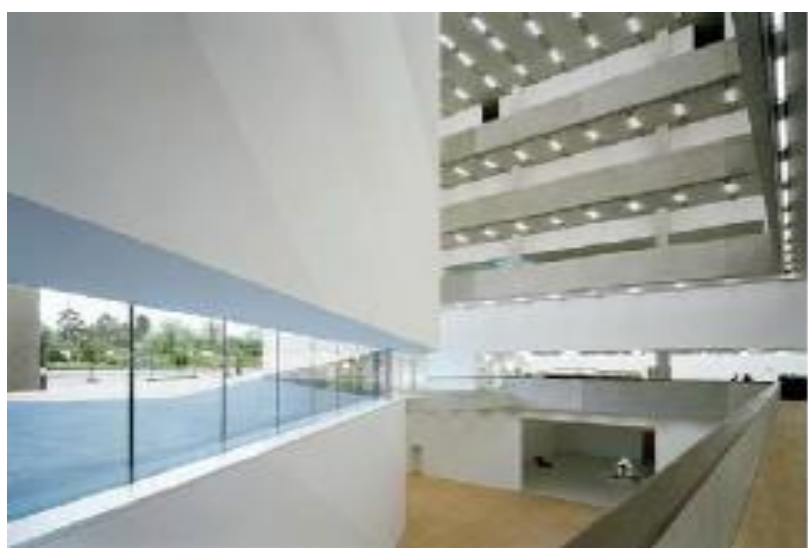

(b)

Plate I; Adaptable Schlaulager (Art Store) in Basel (a)Exterior; (b)Interior, (Source [11])

\subsubsection{Transformability}

Transformability in buildings enables a building to change its shape, space and appearance by the physical modification to its basic components, outer shell or internal surfaces. 


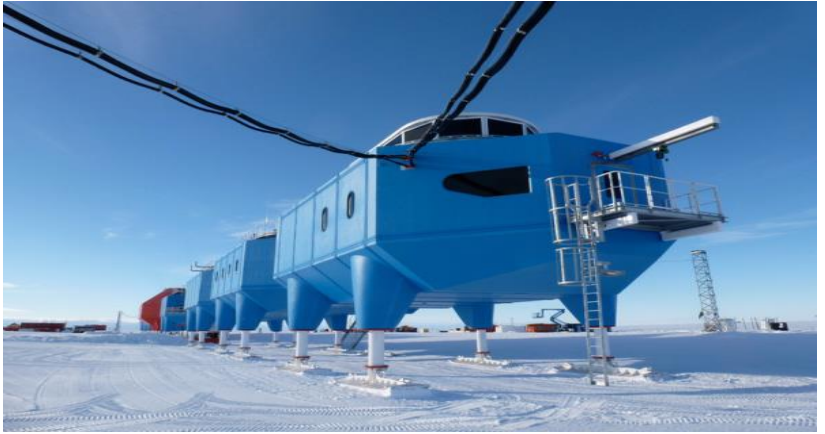

Plate II; Mobile Halley Research Station, Antarctica (Source [11])

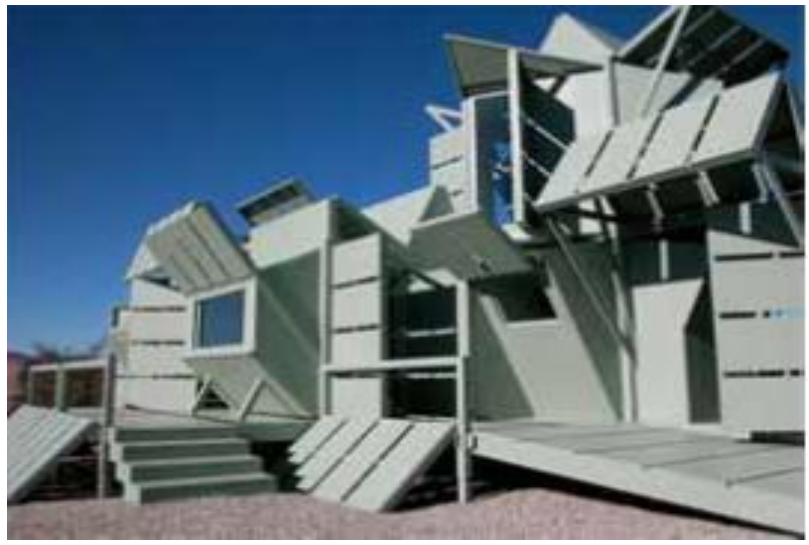

Plate III; Transformable modules M-House by Michael Jentzen(Source [11])

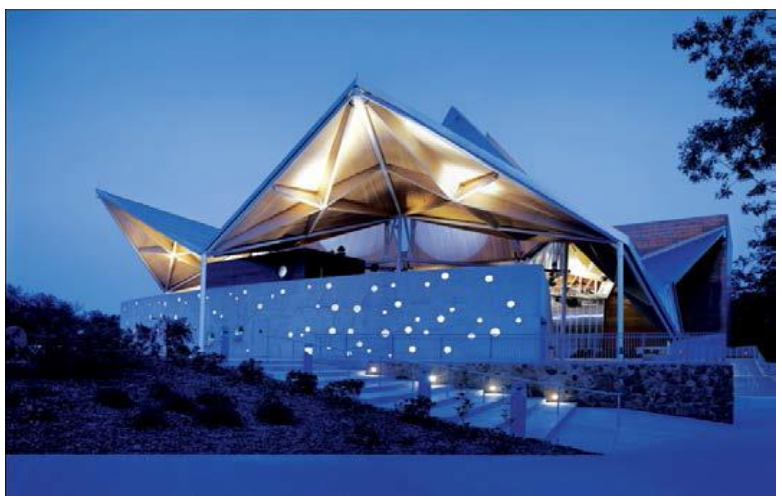

(a)

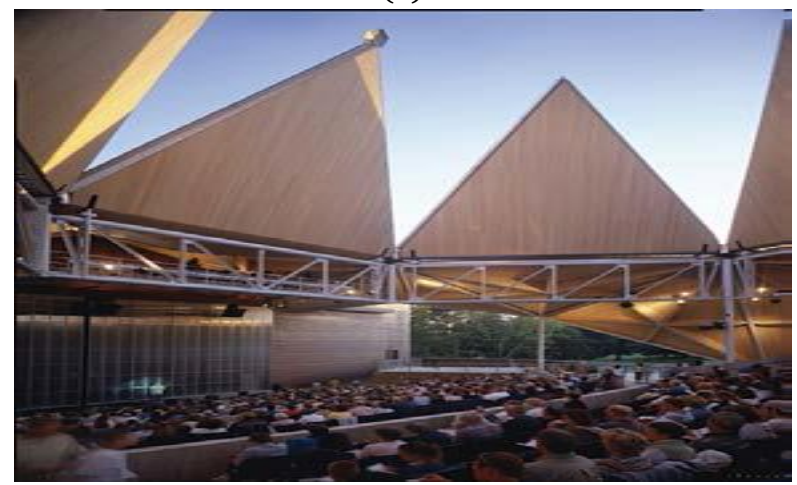

(b)

Plate IV; Starlight Theatre in Illinois (a)closed form (b)opened form (Source [11])
Transformable buildings also have the ability to interact with external environment removing the barrier between inside and outside and respond to climatic situations thereby contributing to sustainability. Components can be opened or closed depending on the purpose and desire of the user. Just as seen in Plate IV showing the Starlight Theatre in Illinois, Plate IV (a) has an enclosed interior while Plate $\mathrm{IV}$ (b) shows as the interior space is transformed into the outside environment.

This transformation enables a building to open, close, contract or expand, making it a critical aspect of flexibility in buildings [7]. The transformation operation can be done manually by disassembling and assembling of different building components, or by mechanical means using a button to control the movement of parts and change of form of a building [11]. An example is the M-House made by Architect Jantzen as seen in Plate III which consists of a series of rectangular panels that are attached in hinges which allows the panels to fold to perform various functions and this structure could be assembled and disassembled.

\subsection{Flexibility of Design Features}

The flexibility of the design components that form a structure is the basic factor that determines how flexible a structure can be. These design features can easily be identified and assembled to enable one realize, create or modify the change requirement. The breaking down of these components that can be disassembled non-destructively from the product as a unit is referred to as modules and each module can be attached, detached, modified, relocated, and replaced easily for upgrading, repair, recycling, or reuse. Demountable structures include modular design which serves as basis for adaptable, mobile and transformable design. The function-based modular design offers flexibility and allows maximum space utilization and functions to satisfy the needs of different groups of users.

\subsubsection{Basic Elements of Design Features}

There are two ways for creating flexible multifunctional spaces; first is by using furniture and secondly by the building construction such as walls and floors [13]. The fact that flexible walls yield space flexibility, modular design also leads to flexible spaces and can be realized by building elements such as walls, furniture, ceilings, doors or windows [14]. 

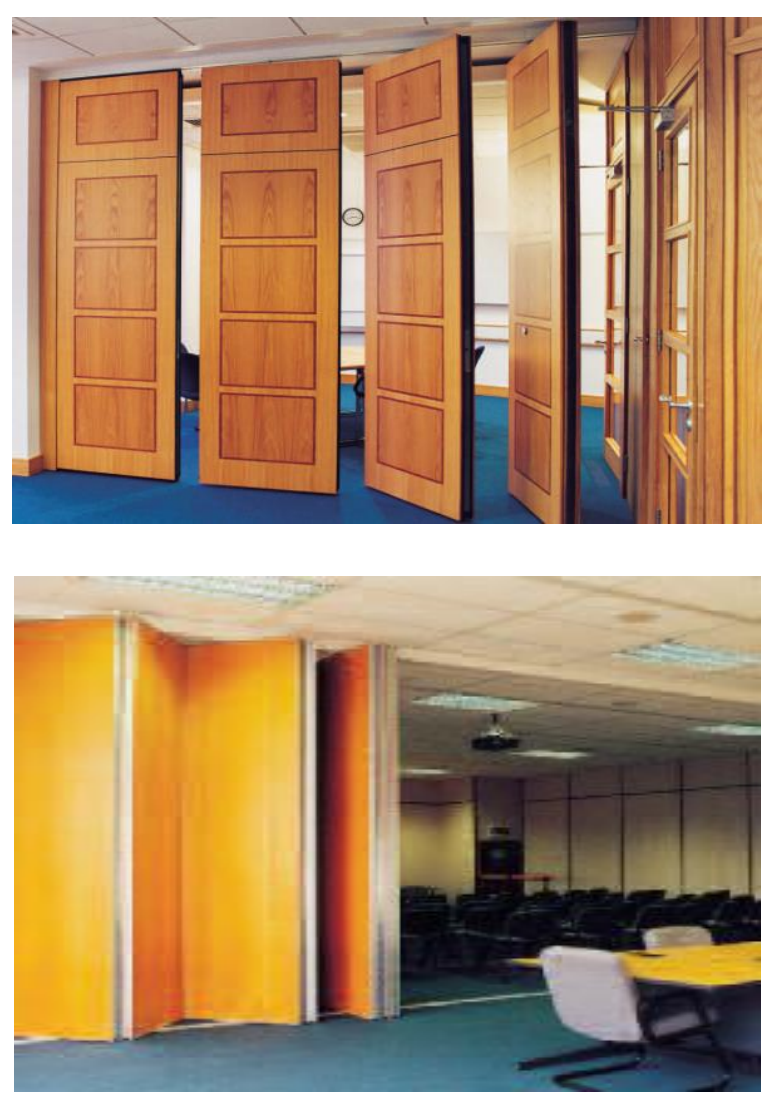

Plate V; Folding and moveable doors used as partitions Source [20]

\subsubsection{Moveable and folding walls}

It is obvious that walls are a necessity in forming flexible interior spaces [14]. Flexible walling systems are made of lightweight materials so that they can be operated easily. Movable walls are specified where functional space demarcation is an essential element within the partition project. Demountable and movable partitions should be used in areas susceptible to future partition rearrangement. Due to their flexibility and cost effectiveness they allow large rooms to be conveniently sub-divided to maximise space utilisation. Compared to traditional wall construction, installation of these partitions is faster and eliminate the cost and mess of cutting and fitting carpet and ceilings around fixed walls. They offer maximum flexibility and reusability to accommodate frequent and quick relocation of work without loss of materials, damage or modification to panels or to adjoining structures such as ceilings, fixed walls and floors. Movable partitions and some demountable partitions are non-progressive allowing the removal of individual panels from any location without disturbing adjoining units. Partition layout should be based on module configuration to allow for maximum reusability. Standard applications include office interiors, meeting rooms, sports and leisure facilities, schools, colleges, churches, conference and function suites, exhibition centres, banks and restaurants. The versatile system can be quickly and easily operated to provide division of room space for meetings, events or activities allowing separate areas to be used without disturbance from each other or opening up to one large room when needed as seen in Plate V.

\subsubsection{Moveable and Retractable Seating}

One of the likely change that can take place in a space is the changes in the function of a space [15]. When static seats are used, it limits the type of function that the space can be used for, thereby making the space less flexible, but when flexible seats are introduced, the space can be rearranged to allow several functions. The most flexible seats that offers multi-functional spaces in performing spaces like in event centres are the retractable seats. Moveable and retractable seating platforms are used in a range of facilities including performing art centres and theatres where there is a requirement for seating areas and floor space to be racked. Due to their flexibility and cost effectiveness they allow large rooms to have different floor levels for multi-purpose use. The benefit of this is that it maximizes use of venue space, allows multiple uses within a facility and provides optimum viewing for the audience. The types include: fixed, recessed, mobile and travelling seating as seen in Figure 2.

\subsubsection{Moveable and Retractable Roofs}

This is a roofing system designed to roll back on tracks so that the interior of the facility is open to the outdoors. The development of these roof structures originated from ancient times when Romans covered their buildings intended for mass events (amphitheatre, theatre, and circus) with foldable awnings. They used simple retractable systems (wooden beams, ropes, canvas) for unfolding the roof above stands and thereby shielding users from a variety of weather conditions [16]. Retractable roof structures are a type of roof structure, which can be completely or partly moved or folded in a short period of time so that the building can be used with an open or closed roof [17]. Retractable roofs are used in residences, restaurants and bars, swim centres, and other facilities wishing to provide an open-air experience at the push of a button. They vary in shape, material and movement and could be categorised based on frequency of opening and closing, structural design, type of movement, size of movable roof elements, and type of moving system. The most generally used folding schemes involve large, rigid elements experiencing 
transformation or rotation and these elements are usually overlapped as seen in Figure 3(a) and 3(b) or folded as seen in Figure 3(c) and 3(d). Figure 3(e) shows a solution in which the retractable cover is a flexible membrane, whose attachments to a cable net can be moved automatically.

\subsection{Overview of Event Centres}

Event centres are major public assembly facilities that attract people of all ages, social backgrounds and interests. It is often regarded to as multi-purpose facility used for activities ranging from sport to entertainment spectator arenas, extending to community and cultural celebrations, religious to motivational venues; the contrast and diversity of events is infinite. Event centres being hubs for cultural and social activities, attract an unpredictable population, thus these centres are to be designed to allow participants and audience to engage in distinct ways.
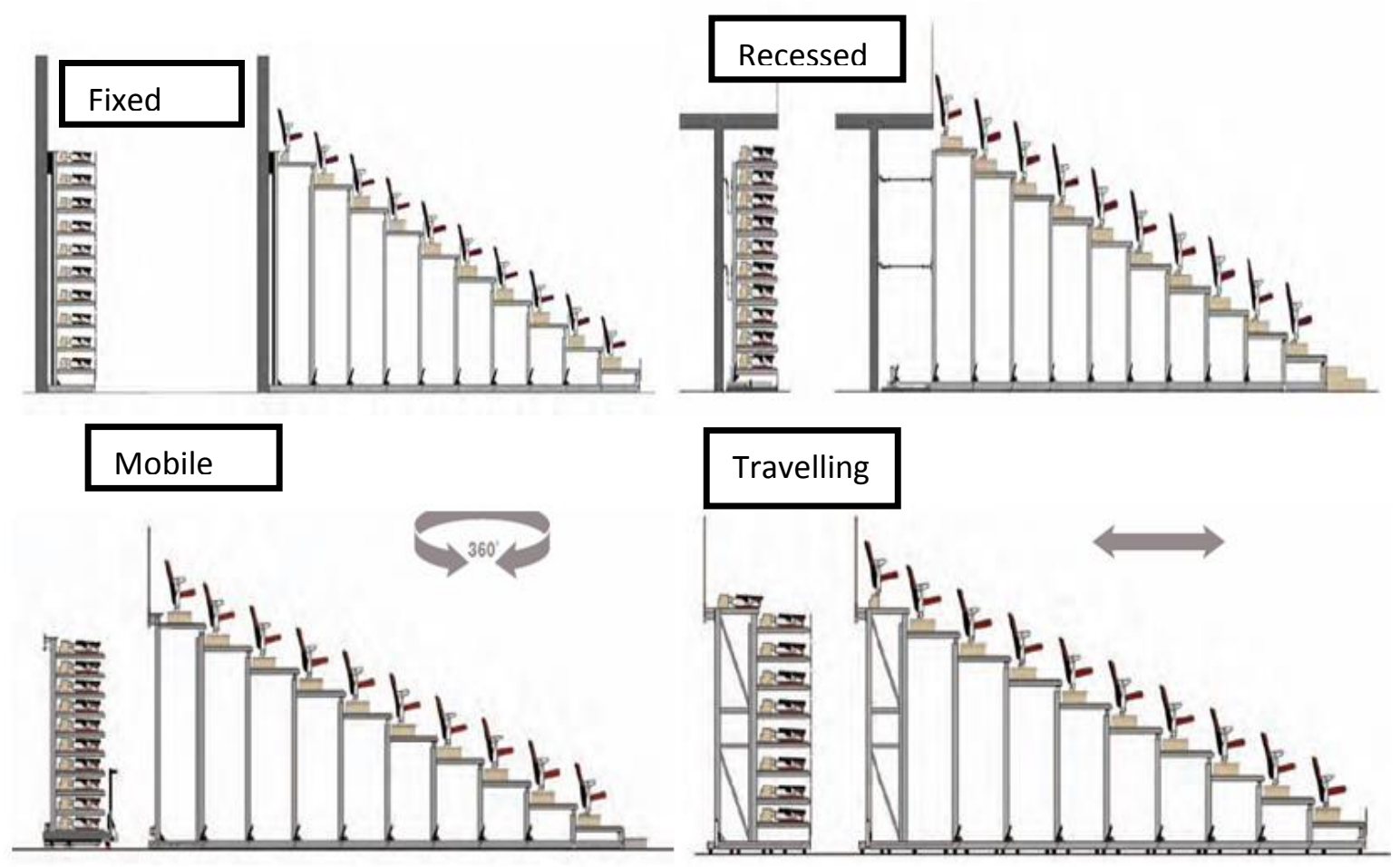

Figure 2: Types of retractable seating (Source [18])

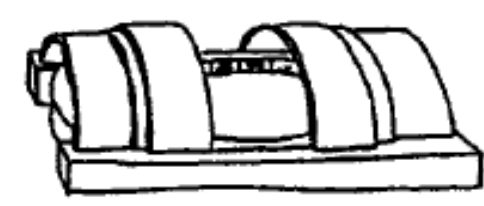

(a)

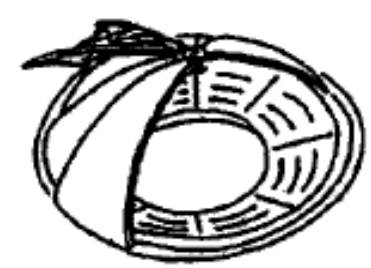

(b)

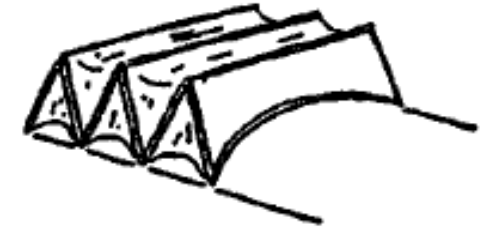

(c)

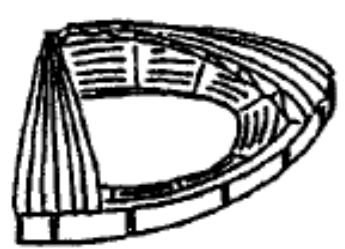

(d)

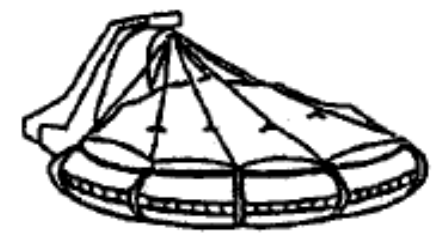

- (e) $-\cdots$

Figure 3: Various opening and closing systems, (Source [17]) 
Event centres are facilities that host varieties of events which change with what society wants, therefore, the need for these facilities to be sustainable to last a long period of time and flexible to adapt to change. Event centres should be able to create a sensation, entertain, aesthetically pleasing and able to accommodate different modes of participation.

\subsubsection{Types of Event Centres \\ Permanent}

A permanent event centre is one which is attached to the ground for a foreseeable future. It is usually planned and designed to remain in a particular location. They are usually constructed on site, or the components could be prefabricated and assembled on site. An example of the temporary event structures in Abuja the Federal Capital Territory of Nigeria, Abuja International Conference Centre shown in Plate VI. It is one of the premier event and wedding venues in the city located in Garki neighbourhood.

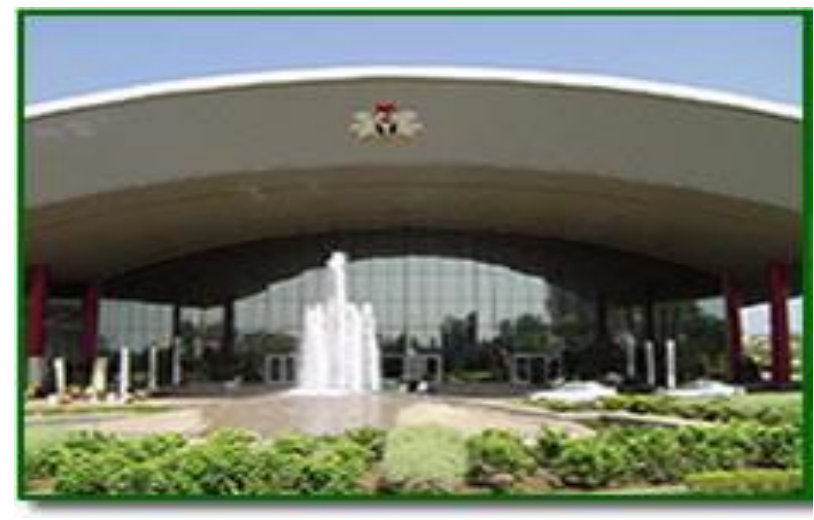

Plate VI; Permanent structure of Abuja International Conference Centre, Abuja (Source. www.abujaicc.com/aicc.htm 2005)

\section{Temporary}

A temporary event centre is one which is not attached to a permanent foundation. It is usually used where there is uncertainty concerning future space requirements or expansion plans. They are usually locatable therefore can be dismantled and removed when they are no longer needed at a convenient time. A temporary event centre is also sustainable because materials are reusable and recyclable and energy usage and cost are kept to a minimum. Plate VII shows a temporary event structure in Abuja the Nations' capital, Cunningham Marquee Event centre which is also among the premier event venues.

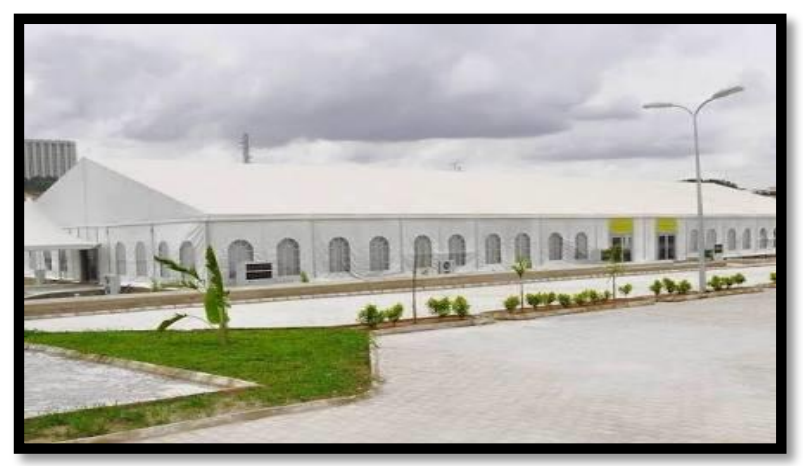

Plate VII; Temporary structure of Cunningham Marquee Event Centre, Abuja. (Source [18])

\section{RESEARCH METHOD}

Observation method of research was employed. Observation method of research involves directly observing and studying a sample of a population. The survey was conducted by directly observing the number of spaces provided in the event centres, with its types of structures and event spaces provided and the flexible design features and approach adopted in the selected event centres. The observation schedule was designed to reveal types of event centre structures and event space available, availability of flexible design elements, types of flexible design features and approach used.

The population was divided into two local government areas which comprise of Minna city namely: Bosso and Chanchaga. Event centres were selected based on the geographical areas which make up Minna city using area random technique as indicated in Table 1. A sample size of ten (10) event centres in Minna, Niger state was drawn from a total population of fifteen (15) event centres within the study area. Minna city have established event centres in the two local government areas that makeup the state capital. However, each of the local government areas have at least one or more of event centres, making the sample size drawn to be $66.6 \%$ of the total population of the study.

Data analysis took the form of simple descriptive statistics and content analysis represented in the form of percentages. Data collated was computed manually and tabulated in Microsoft Excel Spread Sheet Program. Component bar charts and pie charts were used to represent the frequency distribution

\section{RESULTS AND DISCUSSION}

The results obtained from the observation schedule showed that, the number of event venue available at each event centre. This is represented in Table 2 where 
it is observed that $60 \%$ of the population had a single building for event venues, while a total of $40 \%$ had more than a single venue with $20 \%$ having more than four venues for events at their centre. This shows that event centres with a single venue did not provide spaces for different population, whereas those with 4 or more buildings provided various spaces for different population, hence event centres should provide more than one space for events.

In assessing event spaces available, it was observed that a total of $90 \%$ of the event centres studied were indoor spaces, $30 \%$ of this also had outdoor spaces, but $10 \%$ of the population had only outdoor spaces for events as seen in the Table 3. Deduction from the result obtained shows that an event centre design should have provision for both indoor and outdoor events.

The predominant structure used for event centres in Minna as observed is permanent structure which is $60 \%$ of the research population while $30 \%$ had temporary structure and the remaining $10 \%$ had provision for both. Due to the fact that majority had permanent structure, it shows that these event centres are not flexible.

\subsection{Assessment of Design features for flexibility}

As observed from Table 4, 100\% of the population studied had adaptability as their flexible design approach and this is as a result of the use of large open spaces. However, mobility was seen in $40 \%$ of the population, and this was seen where temporary structures were used for the event centres since they can always be disassembled and assembled at another location. The result also shows that none of the sample population had transformability as a flexible design approach. There was no change in their shape, space and appearance; their basic design features such as roofs, floors and walls were not flexible.

From Table 5 it was observed that only $20 \%$ of the population had flexible design elements available, the remaining $80 \%$ had no design features for flexibility.

Also from the Table 6, it can further be seen that, the event centres in the study area, had $10 \%$ moveable walls and the other $10 \%$ retractable roofs; whereas none of the event centres had adjustable floors.

Table 1: Event centres studied categorised into local government areas

\begin{tabular}{|c|c|c|}
\hline S/No. & Name of Event centre & $\begin{array}{l}\text { Local Government } \\
\text { Area }\end{array}$ \\
\hline 1 & $\begin{array}{l}\text { Abdulsalam Youth } \\
\text { Centre }\end{array}$ & Chanchaga \\
\hline 2 & $\begin{array}{l}\text { COE Multipurpose } \\
\text { Square }\end{array}$ & Chanchaga \\
\hline 3 & Nasfah Hotel & Chanchaga \\
\hline 4 & Uk Bello Art \& Culture & Chanchaga \\
\hline 5 & Vagosh Event Centre & Bosso \\
\hline 6 & $\begin{array}{l}\text { Legbo Kutigi Conf. } \\
\text { Centre }\end{array}$ & Bosso \\
\hline 7 & Gidan Matasa & Bosso \\
\hline 8 & $\begin{array}{l}\text { St. Michael Catholic } \\
\text { Hall }\end{array}$ & Bosso \\
\hline 9 & Posh Event Centre & Bosso \\
\hline 10 & Athena Event Arena & Bosso \\
\hline
\end{tabular}

Table 2: Number of event venue available

\begin{tabular}{llcc}
\hline \multirow{2}{*}{ S/No. } & Name of Event centre & \multicolumn{2}{c}{ Number of Event venue } \\
\hline 1 & Abdulsalam Youth Centre & $\checkmark$ & 3 \\
2 & COE Multipurpose Square & $\checkmark$ & $\checkmark$ \\
3 & Nasfah Hotel & & $\checkmark$ \\
4 & Uk Bello Art \& Culture & $\checkmark$ & $\checkmark$ \\
5 & Vagosh Event Centre & & $\checkmark$ \\
6 & Legbo Kutigi Conf. Centre & & \\
7 & Gidan Matasa & & \\
8 & St. Michael Catholic Hall & $\checkmark$ & \\
9 & Posh Event Centre & $\checkmark$ & 1 \\
10 & Athena Event Arena & $\checkmark$ & \\
\hline & Total Available & 6 & \\
\hline
\end{tabular}

Source: Authors' fieldwork, 2017

Table 3: Types of event spaces available

\begin{tabular}{llrc}
\hline \multirow{2}{*}{ S/No. } & \multirow{2}{*}{ Name of Event centre } & \multicolumn{2}{c}{ Event Space available } \\
\cline { 3 - 3 } & Abdulsalam Youth Centre & Outdoor & Indoor \\
\hline
\end{tabular}




\begin{tabular}{llcc}
\hline 2 & COE Multipurpose Square & $\checkmark$ & $\checkmark$ \\
3 & Nasfah Hotel & $\checkmark$ \\
4 & Uk Bello Art \& Culture & & $\checkmark$ \\
5 & Vagosh Event Centre & & $\checkmark$ \\
6 & Legbo Kutigi Conf. Centre & $\checkmark$ & $\checkmark$ \\
7 & Gidan Matasa & & $\checkmark$ \\
8 & St. Michael Catholic Hall & & 6 \\
9 & Posh Event Centre & 1 & $60 \%$ \\
\hline
\end{tabular}

Source: Authors' fieldwork, 2017

Table 4: Type of design approach adopted

\begin{tabular}{clccc}
\hline \multirow{2}{*}{ S/No. } & \multicolumn{2}{c}{ Name of Event centre } & \multicolumn{3}{c}{ Flexible design approach } \\
\cline { 3 - 5 } & Abdulsalam Youth Centre & $\checkmark$ & Mobility & Transformability \\
\hline 2 & COE Multipurpose Square & $\checkmark$ & $*$ & $*$ \\
3 & Nasfah Hotel & $\checkmark$ & $*$ & $*$ \\
4 & Uk Bello Art \& Culture & $\checkmark$ & $\checkmark$ & $*$ \\
5 & Vagosh Event Centre & $\checkmark$ & $*$ & $*$ \\
6 & Legbo Kutigi Conf. Centre & $\checkmark$ & $\checkmark$ & $*$ \\
7 & Gidan Matasa & $\checkmark$ & $\checkmark$ & $*$ \\
8 & St. Michael Catholic Hall & $\checkmark$ & $\checkmark$ & $*$ \\
9 & Posh Event Centre & $\checkmark$ & 4 & 0 \\
10 & Athena Event Arena & $\checkmark$ & $40 \%$ & \\
\hline & Total Available & $100 \%$ &
\end{tabular}

Source: Authors' fieldwork, 2017

Table 5: Flexible design elements available

\begin{tabular}{clcc}
\hline \multirow{2}{*}{ S/No. Name of Event centre } & \multicolumn{2}{c}{ Flexible design element } \\
\cline { 3 - 4 } 1 & Abdulsalam Youth Centre & Available & $\checkmark$ \\
2 & COE Multipurpose Square & $*$ & $\checkmark$ \\
3 & Nasfah Hotel & $*$ & $\checkmark$ \\
4 & Uk Bello Art \& Culture & $\checkmark$ & $\checkmark$ \\
5 & Vagosh Event Centre & $*$ & $\checkmark$ \\
6 & Legbo Kutigi Conf. Centre & $\checkmark$ & $\checkmark$ \\
7 & Gidan Matasa & $*$ & $\checkmark$ \\
8 & St. Michael Catholic Hall & $*$ & $\checkmark$ \\
9 & Posh Event Centre & $*$ & $\checkmark$ \\
10 & Athena Event Arena & $*$ & $80 \%$ \\
\hline
\end{tabular}

Source: Authors' fieldwork, 2017

Table 6: Flexible design elements used

\begin{tabular}{llccc}
\hline \multirow{2}{*}{ S/No. } & \multirow{2}{*}{ Name of Event centre } & \multicolumn{3}{c}{ Types available } \\
\cline { 2 - 5 } & Abdulsalam Youth Centre & Moveable Walls & Retractable roofs & Adjustable floors \\
\hline 1 & COE Multipurpose Square & $*$ & $*$ & $*$ \\
3 & Nasfah Hotel & $*$ & $*$ & $*$ \\
4 & Uk Bello Art \& Culture & $\checkmark$ & $*$ & $*$ \\
5 & Vagosh Event Centre & $*$ & $\checkmark$ & $*$ \\
6 & Legbo Kutigi Conf. Centre & $*$ & $*$ & $*$ \\
7 & Gidan Matasa & $*$ & $*$ & $*$ \\
8 & St. Michael Catholic Hall & $*$ & & $*$ \\
\hline
\end{tabular}




\begin{tabular}{llccc}
\hline \multirow{2}{*}{ S/No. } & \multirow{2}{*}{ Name of Event centre } & \multicolumn{3}{c}{ Types available } \\
\cline { 2 - 5 } & & Moveable Walls & Retractable roofs & Adjustable floors \\
\hline 9 & Posh Event Centre & $*$ & $*$ & $*$ \\
10 & Athena Event Arena & $*$ & $*$ & $*$ \\
\hline & Total Available & 1 & 1 & 0 \\
\hline & Percentage & $10 \%$ & $10 \%$ & $0 \%$ \\
\hline
\end{tabular}

Source: Authors' fieldwork, 2017

Table 6 show that moveable walls were available in one of the centres studied, although this was achieved using curtain to partition or divide spaces. Plate VIII helps visualise the material used for partition, this shows that although the space division was achieved, it did not solve the problem of disturbance to that space.

In the same vein, cotton fabric was used to reduce the total headroom of the event centre, serving as a false roof at one of the event centres studied which was $10 \%$ of the sample population. This was a retractable roof system adopted, but not only that the wrong material was used, it also created a non-functional space at the gallery as seen in Plate IX.

After studying the flexible design elements and approach used at event centres in Minna, Niger state, Figures 6 - 9 showcase designs that incorporated the proper use of these elements to enhance spaces for multipurpose functions. In Figure 6, the three event halls can be combined to form one large space for full capacity events.

In Figure 7, a design that incorporated the use of flexible design elements such as retractable walls for functional space demarcation. The horizontal retractable system can quickly and easily be operated to provide division of room space for events or activities allowing separate areas to be used without disturbance when needed as illustrated in Figure 8.

A design that incorporated the use of retractable seating for where events require flexible seating areas and floor space to be racked. This allows the large event halls to have different floor levels for multipurpose use, thereby maximizing use of the venue space and providing optimum viewing for the audience. This flexible design feature is as seen in Figure 9:
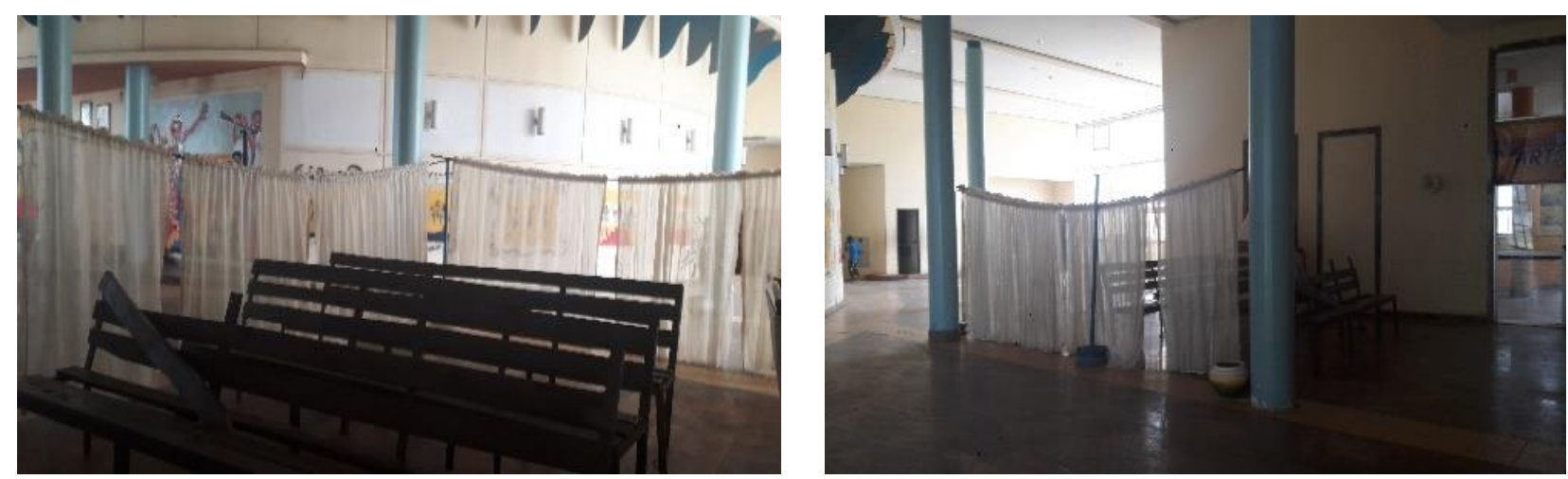

Plate VIII; Curtains used as partition of space at UK Bello Art Theatre, Source: Authors' fieldwork (2017)
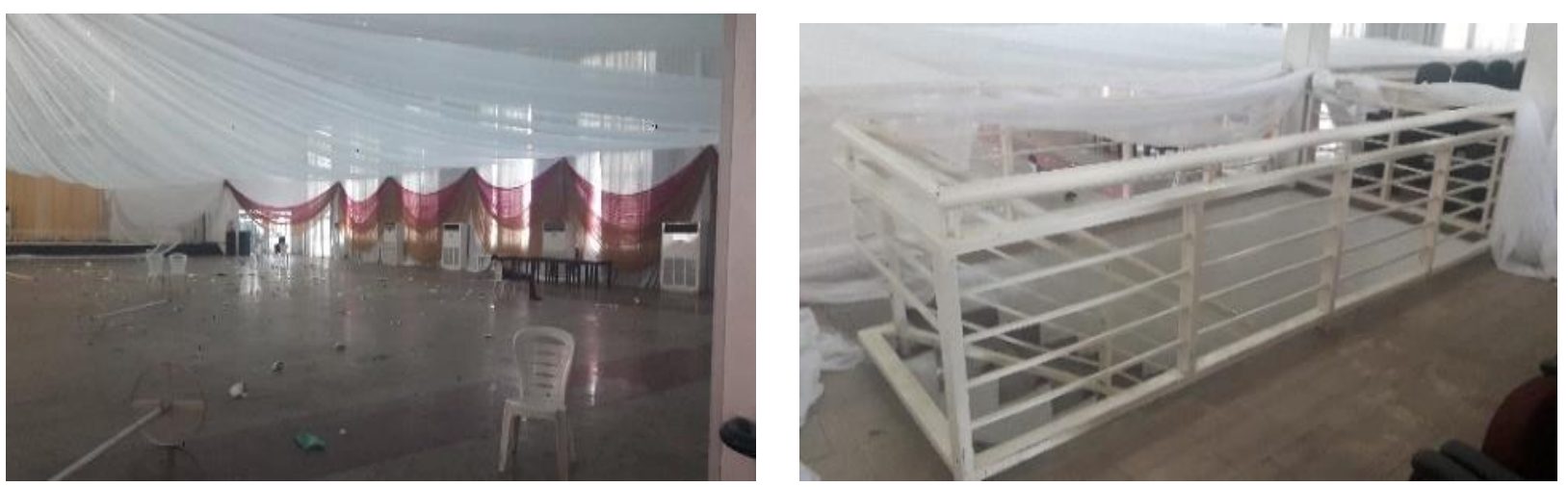

Plate IX; Cotton fabric used as retractable roof to reduce headroom at Legbo Kutigi Hall, Source: Authors' fieldwork (2017) 


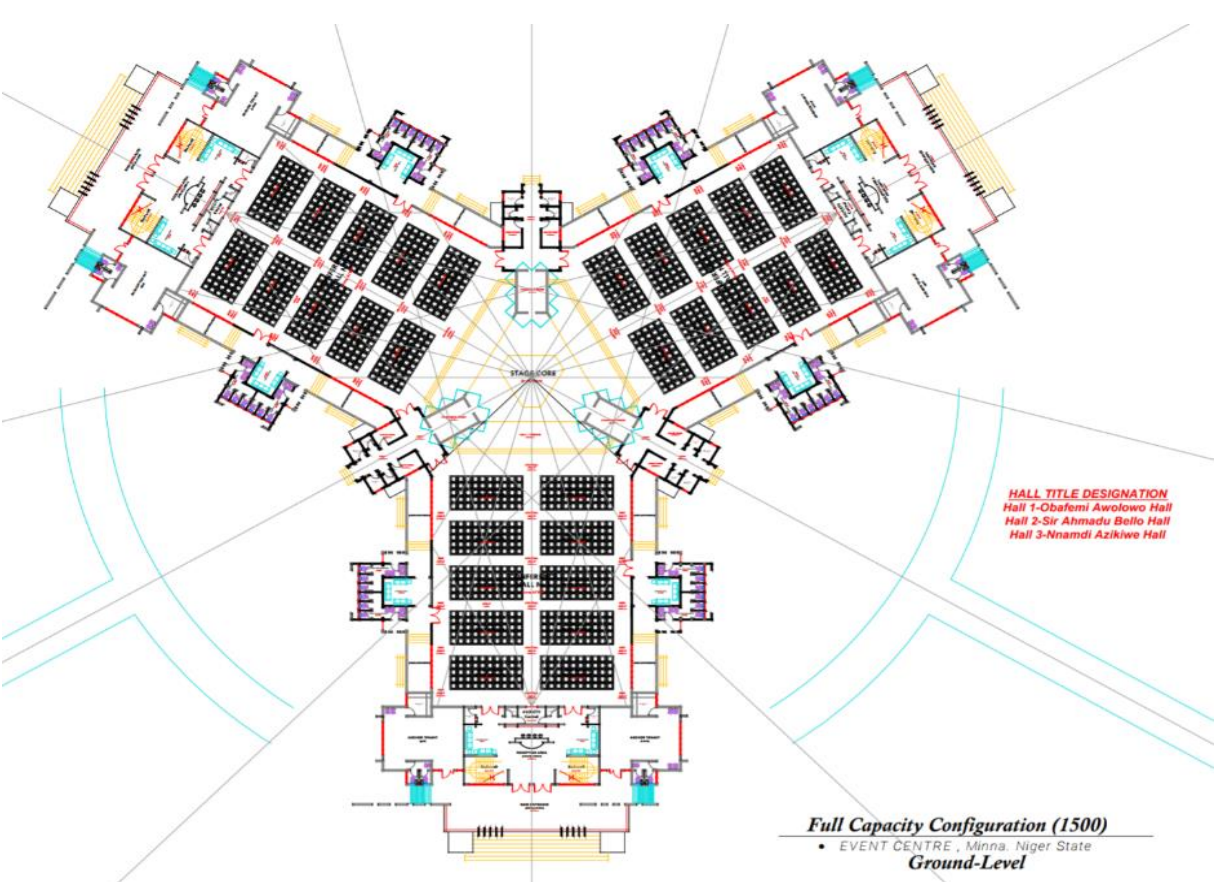

Figure 6: Flexible design elements integrated floor plan of an Event Centre, Source: Authors' work (2017)

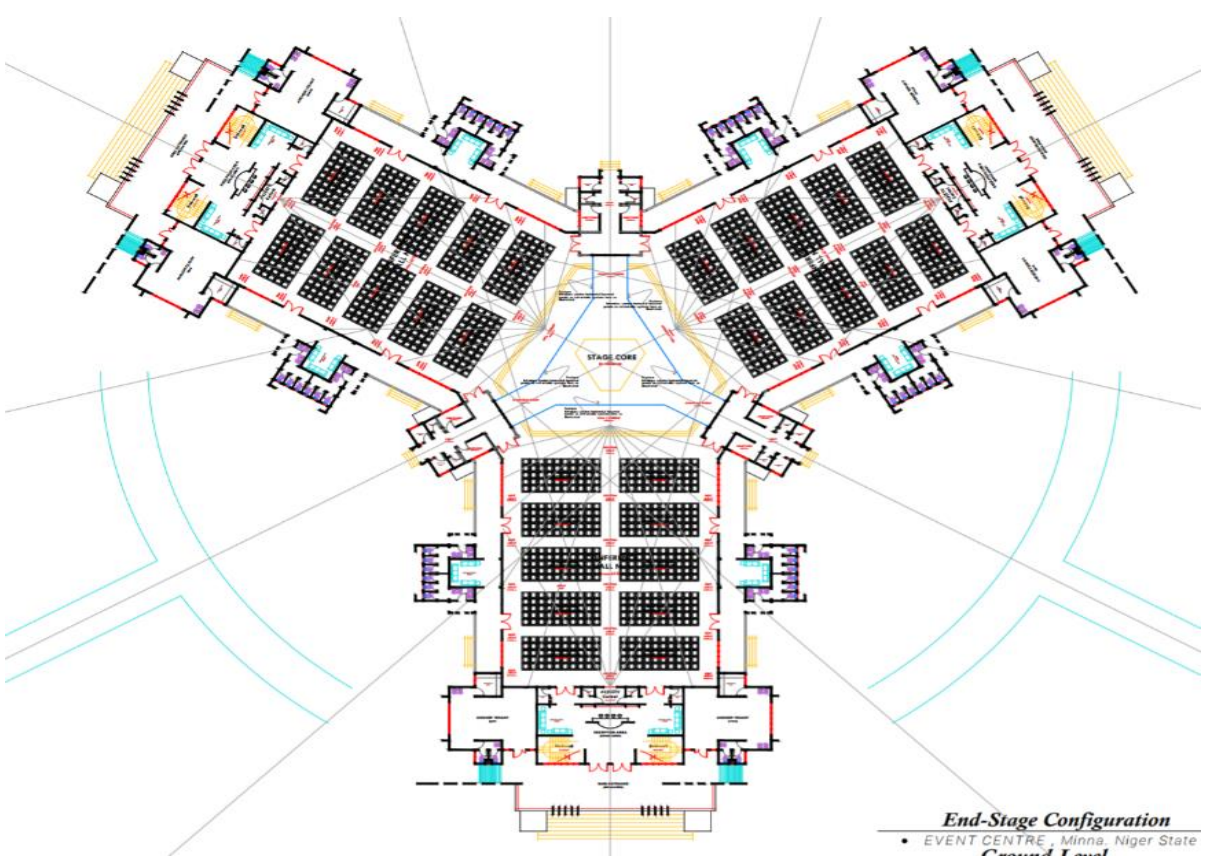

Figure 7: Retractable walls integrated floor plan of an Event Centre, Source: Authors' work (2017)

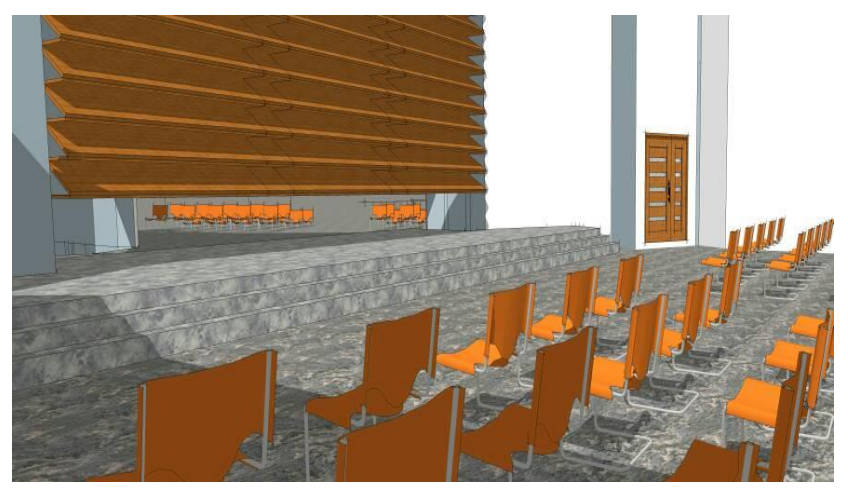

Figure 8: Flexible retractable wall integrated 3D interior of an Event Centre, Source: Authors' work (2017) 


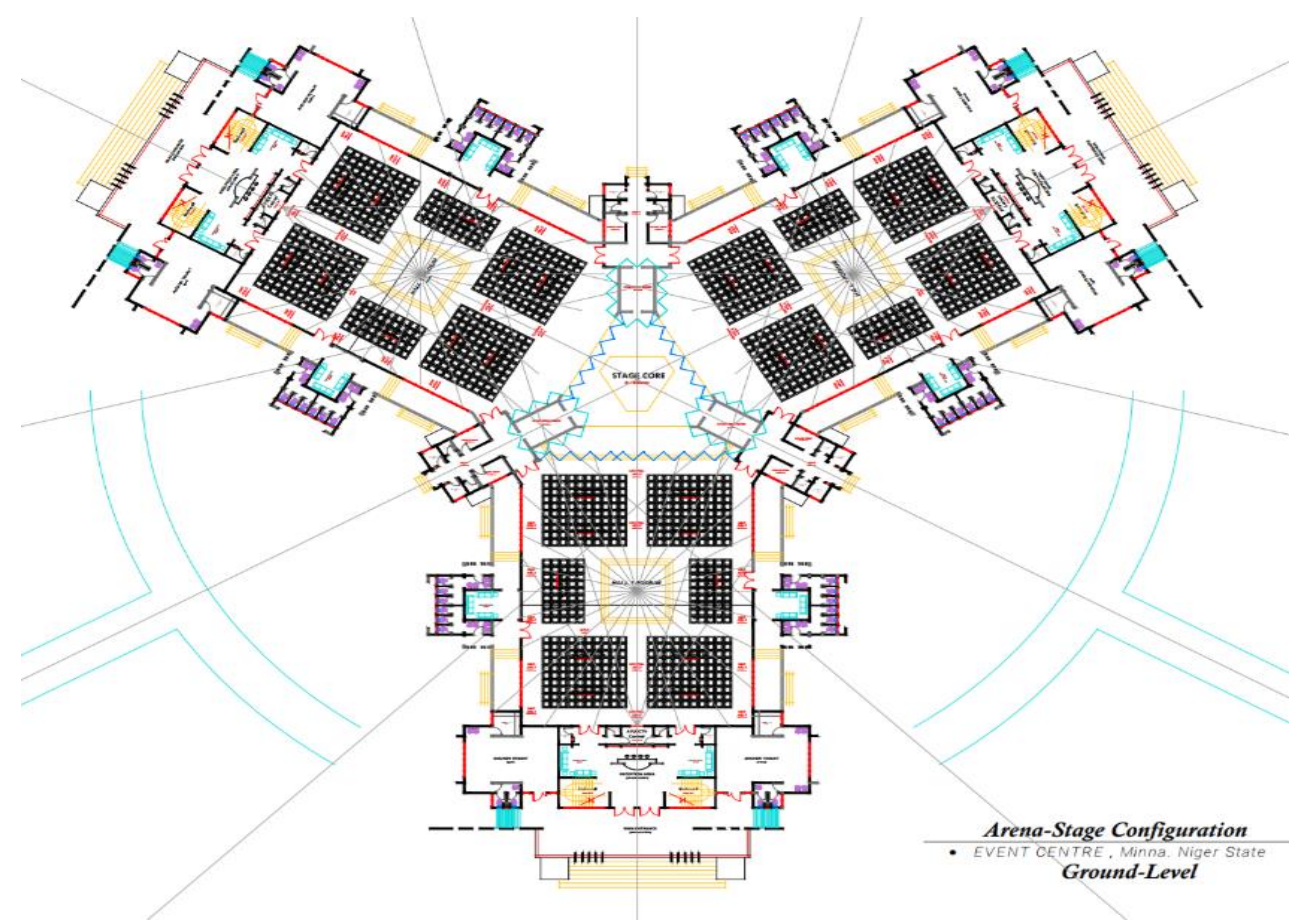

Figure 9: Flexible retractable seating integrated in Arena Stage configuration of an Event Centre, Source: Authors' work (2017)

\section{CONCLUSION AND RECOMMENDATION}

\subsection{Conclusion}

The study has clearly shown that event centres are facilities that attract different categories of persons together, and should be flexible enough to accommodate the needs of different users. In Minna, the study shows that the event centres do not have flexible design features that create or modify a space for change requirement. $60 \%$ which is over half of the sample population of event centres studied had just a single hall for events without providing flexible design features such as moveable walls, retractable roofs or seating in order to make the single hall flexible. Although it was observed that all events centres studied had the open space design which is adaptability, $80 \%$ of event centres in Minna failed to have flexible design features. The $20 \%$ that provided few of these features used materials that partially achieve this purpose making spaces non-functional. Most of these adaptable spaces made use of racked concrete platforms for change in level with fixed seats making the space unable to adapt to changing activities of the event space. Related literatures studied were able to proffer solutions to the flexibility of design features.

\subsection{Recommendation}

In the design of event centres, designers need to recognise the fact that event spaces are multipurpose spaces, therefore it is important to use design features for flexibility such as moveable walls, retractable roofs and sitting should be used in event centre designs to achieve an effective flexible design. Event centres attract an unpredictable population so space flexibility should be considered at all times and adopting all the flexible design approach so that as the users' needs change, the building also changes to meet their needs.

\section{REFERENCES}

[1] A. Ran, ARES Conceptual Framework for software Architecture. In: Jazayeri, M., Ran, A., Van der Linden, F. (Eds), Software Architecture for Product Families Principles and Practice. AddisonWesley, Boston, 2000.

[2] L. Bass, P. Clement, \& R. Kazman, Software Architecture in Practice, Second ed. AddisonWesley, Reading, MA, Boston, 2003.

[3] FEMA 232, Home Builder's Guide to Earthquake Resistant Design and Construction. National Institute of Building Services, Washington DC, 2006.

[4] Masonry Institute of British Columbia (MIBC), Masonry Technical Manual, 3636 4th Avenue East Vancouver, 2015.

[5] S. Mahendra, Re-Quarried: Reclaimed in an urban framework, LAP Lambert Academic Publishing, 2011. 
[6] Architectural Heritage Protection, Guidelines for Planning Authorities: Guidance on Part IV of the Planning and Development ACT 2000, 2004.

[7] R. Kronenburg, Flexible: Architecture that respond to change. Lauren King, London, 2007.

[8] L. Kahn, \& B. Easton, Shelter (p. 176). Shelter Publications, Inc., 1973.

[9] Argency, WFH House, 2012. Retrieved March 06, 2014, from http://arcgency.com/21270/ 452944/gallery/wfh-house

[10] T. Schneider, and J. Till, Flexible Housing: Opportunities and Limits, arq: Architectural Research Quarterly, Vol. 9, 2005.

[11] L. Acharya, Flexible Architecture for Dynamic Societies, Reflection on a Journey from the 20th Century into the Future, Faculty of Humanities, Social Sciences and Education, University of Tromso, 2013.

[12] C. Andrei, Mobiles: The Art of Portable Architecture. Princeton Architectural Press, New York, 2002.

[13] Q. A. Oday, A. S. Omar, \& S. A. Hussien, Impact of Flexibility Principle on the Efficiency of Interior Design. International Transaction Journal of Engineering, Management, \& Applied Sciences \& Technologies, 5(3), 195-212, 2014.

[14] E. Farjami, A. L. Mohammadzadeh, \& A. Taran, Flexibility in Modular Furniture Systems in Open
Offices, Famagusta, North Cyprus. World Academy of Science, Engineering and Technology, International Journal of Social, Management, Economics and Business Engineering 8(5), 12291232.

[15] E. S. Slaughter, Design strategies to increase building flexibility. Building Research \& Information, 29(3), 208-217, 2001

[16] M. Andrej, Typology of Retractable Roof Structures in Stadiums and Sports Halls, University of Ljubljana, 2015. Retrieved from http://www.iu-cg.org/paper/2015/cg03.html.

[17] K. Ishii, Structural Design of Retractable Roof Structures. International Association of Shell and Spatial Structures, WIT Press, Southampton, UK, 2000.

[18] AMG Venues. [Photograph] Retrieved from http://amgvenues.com.ng/venue/Cunninghammarquee/Civic Project. (2017). [19] OzmenMayer B.. [Photograph] Arch 570-Infromal Studies on Housing II. East Mediterranean University, Department of Architecture. Famagusta, 2012.

[20] Syston Doors Movable Acoustic Walls and Sliding Folding Partitions Brochure. Flexible Space Solutions. Albert Street, Syston Leicester. [Photograph]Retrieved from www.syston.com 2017. 